\title{
Asymptotically safe pure gravity as the source of dark energy of the vacuum
}

\author{
Mohamed S. E. Naschie \\ Dept. of Physics, University of Alexandria, Alexandria, Egypt
}

Email address:

Chaossf@aol.com

\section{To cite this article:}

Mohamed S. E. Naschie. Asymptotically Safe Pure Gravity as the Source of Dark Energy of the Vacuum. International Journal of Astrophysics and Space Science. Vol. 2, No. 1, 2014, pp. 12-15. doi: 10.11648/j.ijass.20140201.13

\begin{abstract}
Dark energy can be understood in terms of the asymptotic safety of pure gravity. The present short paper shows how pure gravity, quantum mechanics and fractal spacetime are different aspects of the same reality. Results and conclusions are subsequently reinforced and partially reproduced using different theories related to phase transition, Unruh temperature and 't Hooft renormalization.
\end{abstract}

Keywords: Dark energy, Pure gravity, Asymptotic Safety, Conformal Golden Geometry, Fractal Spacetime, E-infinity Theory, Dimensionless Temperature, Unruh Temperature, 't Hooft Fractal Spacetime, Dimensional Regularization.

\section{Introduction}

In previous work starting from strictly mathematically founded theory based on the new transfinite theory of Woodin and his school [1,2], we have already established that a zero set is the pre-quantum particle $[3,4]$ and that the empty set is the surface of the zero set and represents the quantum wave [3-5]. Translating these mathematical concepts to physics in Kaluza-Klein spacetime we found that ordinary measurable energy is the potential energy of the quantum particles while dark energy which we cannot measure is the kinetic energy of the quantum wave also in five dimensions [3-6]. In the present short letter we would like to argue the case in terms of relatively more conventional concepts and terminology used partially in conformal field theory and string theory [7,9]. When everything is said and done, the picture which arises from the bulk of our work on this subject is that what is labeled pure gravity, i.e. Einstein's general relativity equation in the absence of a matter field is for all practical reasons almost equivalent to a Cantorian empty set [4-6] and consequently we also conjecture that it is effectively equivalent to a topological defect called in the terminology of algebraic topology, texture [10]. Finally to demonstrate the mathematical elegance and truthfulness of the unity of physics and mathematics we reproduce all our present results using the theory of phase transition as well as ' $\mathrm{t}$ Hooft renormalization.

\section{Pure Gravity and Rindler Hyperbolic Horizon}

The number of degrees of freedom is probably the most important aspect of a physical theory. This number decides on the dimensionality of the state space of the system and could be set equal to it under certain conditions [7,9]. For pure gravity theory it is fairly well known that in $\mathrm{d}$ dimensions the field theoretical degrees of freedom is given by [7-9]

$$
D_{d}=d(d-3) / 2
$$

It should be noted that the alignment equations of the Weyl tensor are $(1 / 2) \mathrm{N}(\mathrm{N}-3)$ degrees, i.e. equal to that of pure gravity for $\mathrm{N}=\mathrm{d}$. Thus the number of degrees of freedom of pure gravity is equal to the independent components of the metric. Suppose we are interested in a conformal field theory coupled to a two dimensional pure gravity [7-9]. Inserting $\mathrm{d}=2$ one finds a negative "dimension"

$$
D_{2}=2(2-3) / 2=-1 \text {. }
$$

Regarding $D_{d}$ as a topological Menger-Urysohn dimension [11-18] it is reasonable to look upon the state of pure gravity, i.e. the absence of matter field as an empty set $\mathrm{D}(-1)=-1$. That means $\mathrm{d}=2$ gravity corresponds to an 
empty set. An even more remarkable result comes out of looking at things in a way that if a matter field which we may add to obtain a massive gravity is coupled to our pure gravity then the "degrees of freedom" cancels out and we lose all the local degrees of freedom and end up with something resembling quantum mechanics. Said differently, we obtain a quantum pre-particle modeled with a zero set [3-6]. Thus while pure gravity in two dimensions is an empty set quantum wave, the combination of this empty set with a one dimensional matter field gives us the zero set quantum pre-particle because for $\mathrm{d}=3 \mathrm{D}_{3}=0$.. Remembering again that the Hausdorff dimension of the empty set "wave" is $\phi^{2}$ where $\phi=(\sqrt{5}-1) / 2$ and the corresponding Hausdorff of the zero set is $\phi$, we can understand how the transfinite number $\phi=0.618033989$ entered into our calculations of the ordinary and the dark energy density via the Rindler wedge hyperbolic geometry of black hole-like horizon [17-19]. It is important to note that for $\mathrm{d}=8$ we find $\mathrm{D}=20$, i.e. our degrees of freedom in this case is equal to $R^{(4)}$ of the independent components of the Riemannian tensor in 4 dimensions [12-19].

\section{From Nash Euclidean Embedding to the Fractal Geometry of Quantum Entanglement}

Let us demonstrate where the preceding deceptively elementary considerations can lead us to when we consider the question of smooth Euclidean embedding of all what we mentioned above. We start from Nash embedding of a compact quasi-manifold [15]. This is given by

$$
D(N a s h)=\frac{n}{2}(3 n+11)
$$

Setting $\mathrm{d}=\mathrm{n}$ and equating the two quasi dimensions one finds

$$
\frac{n}{2}(3 n+11)=n(n-3) / 2
$$

That means

$$
\mathrm{N}=-7 \text {. }
$$

This corresponds to a Hausdorff dimension equal to

$$
\mathrm{D}_{\mathrm{c}}^{(-7)}=\phi^{7+1}=\phi^{8} \text {. }
$$

In turn $\phi^{8}$ could be interpreted as a quantum probability of Hardy type entanglement

$$
\mathrm{P}=\phi^{5} \phi^{3}=\mathrm{P}(\mathrm{H} \text { ardy }) \mathrm{P}(\text { vacuum })=\phi^{8}=\frac{1}{47-\phi^{8}}=d_{c}^{(-7)}
$$

Thus as a byproduct of our analysis the paramount importance of quantum entanglement due to zero measure fractals as well as golden mean fat fractals is amply demonstrated [12-20].

\section{Conformal Golden Mean Geometry and Asymptotic Safety}

Now it is time to consider the question of asymptotic safety. It is an obvious and almost trivial fact that fractal spacetime is intrinsically asymptotically safe and there is no place there for ultraviolet catastrophe because the renormalization $1 / 0=1$ is the very basis of the topological unit interval physics used in E-infinity theory. To demonstrate yet again how powerful the intrinsic golden mean binary renormalization is, we give in detail the following derivation of Sommerfield's inverse fine structure constant $\bar{\alpha}_{0}[16,17]$. In a set theoretical sense we can prove that zero is equal to one in our unit interval and this will lead to $\bar{\alpha}_{o}=137.08203393 \simeq 137$. We start from the conformal weight $(\mathrm{CW})$ equation corresponding to a random golden mean fractal geometry $[21,22]$ :

$$
(C W)=\phi+\phi^{3}+\phi^{4}+\left(\phi^{\infty}=0\right) .
$$

Inversing and renormalizing one finds [17]

$(C W)=(1 / \phi)+(1 / \phi)^{3}+(1 / \phi)^{4}+(1 / 0=1)=\overline{\alpha_{0}} / 10$.

Thus taking ten copies of $(\overline{\mathrm{CW}})$ we end with $[21,22]$

$(10) \overline{(C W)}=(10)\left(\overline{\alpha_{0}}\right) / 10=(1 / \phi)(60)+30+9+1=137.0820393$

where $\bar{\alpha}_{1}=60, \bar{\alpha}_{2}=30, \bar{\alpha}_{3}=9, \bar{\alpha}_{4}=1$. The integer part for $\bar{\alpha}_{0}$ is easily written as

$$
\overline{\alpha_{0}}(\text { int eger })=(2)^{7}+(2)^{3}+(2)^{0}=137 .
$$

On the other hand we know that the P-Adic expansion of 137 to the basis 2 is precisely the P-Adic norm [23]

$$
\|\bar{\alpha}=137\|_{2}=\overline{\alpha_{Q}}=1
$$

as shown previously in a somewhat different way in the following three remarkable transformations $[17,23]$

$$
\begin{gathered}
\frac{1}{\phi^{\infty}}=\frac{1}{0} \stackrel{\stackrel{T}{S} \frac{1}{\phi_{0}}=\frac{1}{1}=1,}{10 \rightarrow \overline{\alpha_{0}}} \\
1 \rightarrow \overline{\alpha_{0}} / 10 .
\end{gathered}
$$

From equation (7) to the conclusion that ordinary energy density and dark energy density are an mc2 multiple of $\mathrm{d}_{\mathrm{c}}{ }^{(-6)} / 2=\phi^{5} / 2$ and $1-\left(\phi^{5} / 2\right)=5 \phi^{2} / 2$ respectively is only a trivial distance. More details regarding the above transformation are given in $[12,48]$. In conclusion we may summarize the essence of what we said in one sentence 
which stands in sharp contrast to L. Kronecker: "God created the golden mean transfinite numbers and everything else is the work of man." [24-27]

\section{Critical Dimensionless Temperature and Dark Energy}

The preceding results and conclusion could not only be reinforced but virtually reproduced using the theory of Ising phase transition [29-31] and a quasi-fractal version of Borel resummation which was not known to "t Hooft at the time when he, as a post graduate student, discovered that Borel ingenious method [28,29] of summing divergent series could be used to prove the renormalizability of Yang-Mills theory [32]. In fact it is not particularly difficult to show that the critical dimensionless temperature for one space and one time dimension is almost equal to the golden mean [28]. This proximity is due to numerical rounding errors inherent in the basic model which on removing, clearly shows that the critical temperature is exactly equal to $\phi$ as shown long ago by several authors including the present author in the related context [29]. With this fundamental result at hand, we see that the Unruh dimensionless temperature follows from the quantum entanglement of a single quantum, i.e. "Cantorian" particle as $\mathrm{T}($ Unruh $)=\phi^{4}$ while for two quantum particles we find the famous result of Hardy, namely $\mathrm{P}=\phi^{5}$ [14-20]. Such is the power of mathematical reasons that what appears to be physically completely different fields are in fact one and the same reality albeit a higher reality than the naïve reality. The unreal effectiveness and power of E-infinity theory stems from being anchored in n-categoric theory and Grothendieck K-theory which in effect says that in an inclusive spacetime theory the speed of light, the critical temperature and the Hausdorff dimension of a random Cantor set are all equal $\phi$ $[21,30]$. An inclusive spacetime theory is the opposite of the traditional exclusive theories. In our inclusive theory spacetime is both open and closed, i.e. topologically clopen. It is finite and infinite, i.e. a Poincaré-Beltrami hyperbolic projection which is the reality of the compactified Klein-Penrose fractal tiling with its golden mean $\phi$ proportionality $[21,31]$. In fact in our universe we can give a finite value to diverging expressions via the fractal Borel resummation method. We can go even further and claim that our universe is not only both flat and curved but also static and expanding and all depends on how deep we probe the ultra reality and from which position and view point we look upon it. Our E-infinity K-theory is not simply super symmetric but rather ultra symmetric unifying the opposite in almost the same sense of Hegel's Dialectic and his coincidencia-opposotorium [19-32].

\section{6. 't Hooft Fractal Spacetime and the Cantorian Spacetime Theory}

In the preceding section we alluded to ' $t$ Hooft dimensional regularization theory [32] and its intimate connection to our Cantorian spacetime [32]. In fact dimensional regularization implies Cantorian fractal spacetime. The fundamental equation of ' $t$ Hooft $D=4-\varepsilon$ and $\varepsilon \rightarrow 0$ determines the exact dark energy density by simply not letting $\varepsilon$ become zero but a very small quantity instead equal to twice that of Hardy's quantum entanglement and then divide by 4 . That means for $\varepsilon=2 \phi^{5}=\mathrm{k}$ one finds $\mathrm{E}$ (dark) equal $95.5 \%$. The same philosophy could be extended to a fractal Kaluza-Klein space and one finds $\mathrm{E}$ (dark) percentage of $95.5 \%$ equal to the ratio of $\mathrm{D}=5$ to $\mathrm{D}($ fractal $)=5+\phi^{3}=5.236067977$.

Finally we note that we could reach all these conclusions using yet again another view point due to Wheeler's boundary of a boundary principle and its extension to the no boundary proposal by Hartle and Hawking but this will be given in a future publication.

\section{Conclusion}

Gravity and quantum mechanics are united in the true real vacuum represented by the zero set and the empty set of Cantorian-fractal spacetime [5]. The richness of the pure gravity of Einstein's equation led the way to an asymptotically safe theory in a transfinite setting. The final result is a deeper understanding of dark energy and transfinite-fractal numbers as reality [12-27]. One of the main aims of the present work was to demonstrate not only the unity of physics but also that of physics and mathematics. To this end we reconsidered and reproduced the main results and conclusions using completely different theories due to Wilson, Unruh, 't Hooft and others.

\section{References}

[1] W. Woodin, Strong axioms of infinity and the search for V. In Proceedings of the Int. Congress of Mathemetics, Hyderabad 2010, Vol. 1. Plenary lectures and ceremonies. Editor Rajendra Bhatia, Hidustan Book Co., New Delhi, 2010, pp. 504-530.

[2] R. Elwes, Ultimate Logic. New Scientist, 30 July 2011, pp. 30-33.

[3] M.S. El Naschie, A resolution of cosmic dark energy via a quantum entanglement relativity theory. J. Quantum Info. Sci., 3(1), 2013, pp. 23-26.

[4] M.S. El Naschie, The fractal geometric origin of quantum mechanics from Hardy's quantum entanglement. Journal of E-infinity \& Complexity Theory in High Energy Phys. \& Eng., 1(1), 2011, pp. 1-7.

[5] M.S. El Naschie, Topological-geometrical and physical interpretation of the dark energy of the cosmos as a 'halo' energy of the Schrödinger quantum wave. J. Modern Phys., 4(5), 2013, pp. 591-596.

[6] M.S. El Naschie, Dark energy from Kaluza-Klein space time and Noether's theorem via Lagrangian multiplier method. J. of Mod. Phys., Vol. 4, No. 6, 2013, pp. 757-760. 
[7] M. Kaku, Introduction to Superstrings and M-Theory. Springer, New York, 1999.

[8] D. Kutasov and N. Seiberg, Number of degrees of freedom, density of states and tachyons in string theory and CFT. Nucl. Phys. B, 358(3), 1991, pp. 600-618.

[9] M.J. Duff, The World in Eleven Dimensions. Inst. of Phys. Publications, Bristol, 1999.

[10] A. Vilenkin, and E.S. Shellard, Cosmic Strings and Other Topological Defects. Cambridge University Press, Cambridge, 1994.

[11] H. Lehning, Dynamics of typical continuous functions. Proc. of the American Math. Soc., 123(6), 1995, pp. 1703-1707.

[12] M.S. El Naschie, The theory of Cantorian spacetime and high energy particle physics (an informal review). Chaos, Solitons \& Fractals, 41(5), 2009, pp. 2635 - 2646.

[13] S.I. Nada, Density manifolds, geometric measures and high energy physics in transfinite dimensions. Chaos, Solitons \& Fractals, 42, 2009, pp. 1539-1547.

[14] M.S. El Naschie, A unified Newtonian-relativistic quantum resolution of the supposedly missing dark energy of the cosmos and the constancy of the speed of light. Int. J. Modern Nonlinear Sci. \& Application., 2(1), 2013, pp. 43-54.

[15] M.S. El Naschie, Nash embedding of Witten's M-theory and the Hawking-Hartle quantum wave of dark energy. J. Mod. Phys., 4, 2013, pp. 1417-1428.

[16] M.S. El Naschie, Exact non-perturbative derivation of gravity's $\mathrm{G}_{4}$ fine structure constant, the mass of the Higgs and elementary black holes. Chaos, Solitons \& Fractals, 37(2), 2008, pp. 346-359.

[17] M.A. Helal, L. Marek-Crnjac and Ji-Huan He, The three page guide to the most important results of M.S. El Naschie's research in E-infinity and quantum physics and cosmology. Open J. Microphys., 3(4), 2013, pp. 141-145.

[18] M.S. El Naschie, Experimentally based theoretical arguments that Unruh's temperature, Hawking's vacuum fluctuation and Rindler's wedge are physically real. American J. Mod. Phys., 2(6), 2013, pp. 357-361.

[19] M.S. El Naschie, Pinched material Einstein spacetime produces accelerated cosmic expansion. Int. J. Astron. \& Astrophys. 4(1), 2014 pp. 80-90.
[20] M.S. El Naschie, A Rindler-KAM spacetime geometry and scaling the Planck scale solves quantum relativity and explains dark energy. Int. J. of Astronomy and Astrophysics, 3(4), 2013, pp. 483-493.

[21] M.S. El Naschie, The theory of Cantorian spacetime and high energy particle physics (an informal review). Chaos, Solitons \& Fractals, 41(5), 2009, pp. 2635 - 2646.

[22] Y. Nutku, C. Saclioglu and T. Turgut (Editors): Conformal Field Theory. Perseus Publishing, New York, 2000.

[23] M.S. El Naschie, P-Adic unification of the fundamental forces and the standard model. Chaos, Solitons \& Fractals, 38 2008, pp. 1011-1012.

[24] P. Mason, Quantum Glory. XP Publishing, Arizona, USA 2010.

[25] Leonard M Wapner,.The Pea and The Sun. A.K. Peters Ltd. Wellesley, MA 02482, 2005.

[26] Valeri A. Rubakov, Large and infinite extra dimensions. Physics-Uspekhi, 44(9), 2001, pp. 871-893.

[27] L. Marek-Crnjac, Cantorian Space-Time Theory - The Physics of Empty Sets in Connection With Quantum Entanglement and Dark Energy. Lambert Academic Publishing, Saarbrücken, Germany, 2013.

[28] A.M. Shalaby, The fractal self-similar-Borel algorithm for the effective potential of the scalar field theory in one time plus one space dimension. Chaos, Solitons \& Fractals, 34, 2007, pp. 709-716.

[29] M.S. El Naschie, Renormalization approach to the dimension of diffusion in Cantorian space. Appl. Math. Lett., 8(1), 1995, pp. 59-63.

[30] I. Gottlieb, M. Agop and M. Jarcau, El Naschie's Cantorian space-time and general relativity by means of Barbilian's group: A Cantorian axiomatic model of spacetime. Chaos, Solitons \& Fractals, 19(4), 2004, pp. 705-730.

[31] M.S. El Naschie, The meta energy of dark energy. Open J. of Philosophy, 2014. In press.

[32] M.S. El Naschie, Logarithmic running of 't Hooft-Polyakov monopole to dark energy. Int. J. High Energy Phys., 1(1), 2014, pp. 1-5. 